\section{Dentists in the US should be integrated into the hospital team}

R. P. Nalliah, ${ }^{* 1}$ V. Allareddy ${ }^{2}$ and V. Allareddy ${ }^{3}$
IN BRIEF
- Explains the burden of dental disease and the magnitude of hospital resources used to manage dental problems in hospitals instead of dental offices in the United States.
- Proffers reasons why dental patients sometimes seek care in hospitals in the United States.
- Justifies why oral health should be integrated into general medicine rather than continue operating in silos.

Few dentists play integrated roles within hospital settings and few hospital emergency rooms have dentists. Existing research shows that only 67\% of ER visits for dental problems are managed appropriately. Every year Americans die in hospital after being admitted because of dental disease and a recent study found that 20 people died after such hospitalisations in the United States in 2008. Authors of the current correspondence have conducted extensive research on characteristics of emergency room visits and hospitalisations due to various dental conditions using a nationwide dataset for the United States. This opinion article is intended to provoke discussion about the restructuring of hospitals to integrate oral health professionals into the hospital team.

\section{INTRODUCTION}

Historically, oral health and general medicine have been detached. However, both professions are based on evidencebased, prevention-oriented practice that seeks to alleviate suffering. Physicians are under a lot of pressure with the demands of modern medicine in an environment with limited resources and limited time. The dental profession has positioned itself in a particular niche in the healthcare system where it serves the public through small and medium sized private practices. Few dentists play supporting roles within hospital settings and few hospital emergency rooms have dentists. This article is intended to provoke discussion about the restructuring of hospitals to integrate oral health professionals into the hospital team.

Authors of the current article have conducted extensive research on the characteristics of hospital emergency room (ER) visits made for various conditions, including dental conditions, using a nationwide dataset for

Instructor, Department of Global Health, Harvard School of Dental Medicine, 188 Longwood Avenue, Boston, MA 02115, United States; ${ }^{2}$ Assistant Professor, Department of Paediatric Critical Care, Case Western Reserve University School of Medicine, 11100 Euclid Ave, Cleveland, OH 441065, United States: ${ }^{3}$ Associate Professor, Department of Orthodontics, College of Dentistry - The University of lowa, 801 Newton Road, lowa City, IA 52242, United States

${ }^{*}$ Correspondence to: Romesh P. Nalliah

Email:romesh_nalliah@hsdm.harvard.edu;

Tel: +1 6178172301

\section{Refereed Paper}

Accepted 10 January 2014

DOI: 10.1038/sj.bdj.2014.245

${ }^{\circ}$ British Dental Journal 2014; 216: 391-392 the United States. Findings from these studies identify comorbidities and characteristics of high risk populations. Opinions presented here are based on the expertise gained from this work.

\section{THE ARGUMENT FOR INTEGRATION IN THE HOSPITAL TEAM}

The dental profession is in a unique corner of the healthcare system. Although dentists perform anaesthesia and minor surgical procedures on most of their patients, they are often regarded as a business first and as a healthcare professional second. This is reflected in the attitudes of many patients who regard oral health as a luxury and, somehow, less important than general health. Similarly, most US insurance plans have reduced oral health to an optional extra. However, existing research highlights a growing volume of evidence demonstrating associations between systemic and oral conditions. ${ }^{1,2}$ Moreover, research shows that physicians are not well informed about these interactions. ${ }^{3}$

There is already evidence for the existence of a bidirectional relationship between diabetes and oral health. ${ }^{2}$ Subsequently, providing diabetic care without integrating oral healthcare is an access to care issue. Physicians and insurance companies who facilitate concurrent dental care to their diabetic patients will improve their diabetic patients' outcomes.

It is the position of the authors of this paper that oral health is a crucial part of general health. Every year people die in hospitals after being admitted because of dental disease and a recent study found that
20 people died after such hospitalisations in the United States in 2008. ${ }^{4}$ It is clear that oral health expertise is necessary in hospitals and hospital emergency rooms (ER). Something that remains unclear is the role of dentists in hospital settings.

Our research has demonstrated that 400,000 ER visits were made in one year in the United States due to pulpal and periapical lesions, ${ }^{5}$ with nationwide hospital charges of $\$ 163$ million. A total of 6,000 patients had to be hospitalised and incurred mean hospital charges of $\$ 14,000$ each. This also resulted in 17,000 hospitalisation days across the United States. Another study considered patients hospitalised due to dental conditions and found that nationwide hospital charges for the year of the study was $\$ 1.2$ billion dollars. ${ }^{4}$ This study showed that there were over 50,000 hospitalisations due to dental conditions across the United States in the year of the study and over 170,000 hospitalisation days due to dental conditions. Clearly, every year in the United States oral disease leads to the use of a large amount of hospital resources. The total burden of dental disease on the community is grossly underestimated by our series of studies because our dataset does not capture any information about post-discharge resource use like prescription medication, rehabilitation and follow-up care.

Recent research has shown that there are over 50,000 ER visits each year that are associated with dental complaints made by those who are aged 60 years or older. ${ }^{6}$ Importantly, this study also showed that these older adults ER visits resulted in 
higher hospital charges than the general population, which indicates a larger use of hospital resources. This may be attributable to multiple medical conditions that older patients may have in addition to their dental complaint. It is important to note that the United States has an aging population and the need to effectively manage dental-related visits in hospital settings by older adults is likely to be a growing problem. Consideration should be given to integrating oral health professionals into the ER team to be able to manage these dental complaints quickly and efficiently. With the correct resources a qualified dentist can manage these dental complaints quickly and simply. However, few hospitals have dentists and even fewer have appropriate dental equipment to enable a dentist to practice. It should also be noted that existing research shows that only 67\% of ER visits for dental problems are managed appropriately. ${ }^{7}$ Moreover, about half of these visits were managed with prescriptions and the patients still needed to see a dentist.

In the United States dental services have traditionally been delivered through private dental offices. However, our series of studies have shown that a large number of individuals present to hospitals with dental complaints. The reason for their hospital attendance may be due to a lack of access to a dental provider or because their condition is so severe it warrants a hospital visit. Clearly, oral health-related hospital visits and hospitalisations utilise a considerable amount of hospital resources each year and require expert management by oral health professionals - pain killers or antibiotics won't do. Due to the volume of dental-related ER visits, the large use of hospital resources to manage these conditions and the increasing volume of research that shows connections between oral and systemic disease, there is a growing need to integrate dental professionals into the hospital team and integrate dental care into holistic medicine.

The results of our series of studies add to existing knowledge about the importance of considering oral health as a part of systemic health in managing patients, making healthcare policy decisions, designing insurance plans or even designing curriculums for healthcare fields including medicine and dentistry.

\section{CONCLUSION}

Research has shown that a large number of patients with dental complaints present to the hospital emergency department in a developed country. Many are hospitalised and some die. It is necessary to integrate dentists into the hospital team to prevent morbidity, reduce hospital charges and more efficiently use hospital physician resources to manage overall health of patients in hospitals.

1. Fisher M A, Borgnakke W S, Taylor G W. Periodontal disease as a risk marker in coronary heart disease and chronic kidney disease. Curr Opin Nephrol Hypertens 2010; 19: 519-526.

2. Stojanović N, Krunić J, Cicmil S, Vukotić O. Oral health status in patients with diabetes mellitus type 2 in relation to metabolic control of the disease. Srp Arh Celok Lek 2010; 138: 420-424.

3. Al-Khabbaz A K, Al-Shammari K F, Al-Saleh $\mathrm{N}$ A. Knowledge about the association between periodontal diseases and diabetes mellitus: contrasting dentists and physicians. J Periodontol 2011; 82: 360-366

4. Allareddy V, Kim M K, Kim S et al. Hospitalizations primarily attributed to dental conditions in the United States in 2008. Oral Surg Oral Med Oral Pathol Oral Radiol 2012; 114: 333-337.

5. Nalliah R P, Allareddy V, Elangovan S et al. Hospital emergency department visits attributed to pulpal and periapical disease in the United States in 2006. J Endod 2011; 37: 6-9.

6. Nalliah R P, Lee M K, Rampa S, Whitcomb M, Allareddy V, Allareddy V. Hospital-based ED visits with dental conditions among geriatric patients. J Mass Dent Soc 2013; 62: 24-28.

7. Tulip D E, Palmer N O. A retrospective investigation of the clinical management of patients attending an out of hours dental clinic in Merseyside under the new NHS dental contract. Br Dent J 2008; 205: 659-664.. 